\title{
Early urinary tract infection after spinal cord injury: a retrospective inpatient cohort study
}

\author{
Louise M. Goodes ${ }^{1} \cdot$ Gabrielle K. King ${ }^{1} \cdot$ Alethea Rea $^{2} \cdot$ Kevin Murray $\mathbb{1}^{3} \cdot$ Peter Boan $^{4} \cdot$ Anne Watts $^{5} \cdot$ Jen Bardsley $^{5}$. \\ Carly Hartshorn ${ }^{6} \cdot$ Jeffrey Thavaseelan ${ }^{6} \cdot$ Matthew Rawlins $\mathbb{D}^{7} \cdot$ James A. Brock ${ }^{8} \cdot$ Sarah A. Dunlop ${ }^{1}$
}

Received: 10 April 2019 / Revised: 5 July 2019 / Accepted: 10 July 2019 / Published online: 6 August 2019

(c) The Author(s), under exclusive licence to International Spinal Cord Society 2019

\begin{abstract}
Study design Retrospective audit.

Objectives Examine factors associated with urinary tract infection (UTI), UTI incidence and impact on hospital length of stay (LOS) in new, inpatient adult traumatic spinal cord injury (SCI).

Setting Western Australian Hospitals managing SCI patients.

Methods Data on UTIs, bladder management and LOS were obtained from hospital databases and medical records over 26 months. Adherence to staff-administered intermittent catheterisation (staff-IC) was determined from fluid balance charts. Results Across the cohort ( $n=70$ ) UTI rate was 1.1 starts/100 days; UTI by multi-resistant organisms 0.1/100 days. Having $\geq 1$ UTIs compared with none and longer duration of initial urethral indwelling catheterisation (IDC) were associated with longer LOS ( $p$-values $<0.001)$. For patients with $\geq 1$ UTIs $(n=43 / 70)$, longer duration of initial IDC was associated with shorter time to first UTI ( 1 standard deviation longer [SD, 45.0 days], hazard ratio (HR): $0.7,95 \%$ confidence interval [CI] 0.5-1.0, $p$-value 0.044). In turn, shorter time to first UTI was associated with higher UTI rate (1 SD shorter [30.7 days], rate ratio (RR): $1.32,95 \%$ CI 1.0-1.7, $p$-value 0.039$)$. During staff-IC periods $(n=38 / 70)$, protocols were followed $(85.7 \% \leq 6 \mathrm{~h}$ apart, $96.1 \%<8 \mathrm{~h}$ ), but $26 \%$ of IC volumes exceeded $500 \mathrm{~mL}$; occasional volumes $>800 \mathrm{~mL}$ and interruptions requiring temporary IDC were associated with higher UTI rates the following week (odds ratios (ORs): 1.6, 95\%CI 1.1-2.3, $p$-value 0.009 ; and 3.9, 95\%CI 2.6-5.9, $p$-value $<0.001$ respectively).

Conclusions Reducing initial IDC duration and limiting staff-IC volumes could be investigated to possibly reduce inpatient UTIs and LOS.

Sponsorship None
\end{abstract}

\section{Introduction}

The effects of spinal cord injury (SCI) on the urinary bladder are immediate with both altered voiding dynamics and pathogenic mechanisms including bladder ischaemia, hypoperfusion, abnormal microbiological architecture, damage to the urothelial barrier and dysregulated inflammatory responses rendering patients susceptible to urinary tract infection (UTI) [1]. Following SCI, genitourinary

Supplementary information The online version of this article (https:// doi.org/10.1038/s41393-019-0337-6) contains supplementary material, which is available to authorised users.

Sarah A. Dunlop

sarah.dunlop@uwa.edu.au

Extended author information available on the last page of the article. diseases, including UTI, result in significant long-term morbidity and are the leading cause of rehospitalisation [2-4] with urinary tract morbidity being the second leading cause of death [5]. A retrospective audit of 815 patients in Western Australia (WA) with traumatic SCI between 1971 and 2011 identified genitourinary complications as the most frequent $(34.5 \%)$ cause for hospital readmission [6]. Patients themselves rank bladder and bowel function amongst their top health priorities [7] with urinary incontinence and UTI listed among the most distressing complaints affecting quality of life [8].

Initial neurogenic bladder management is directed at preventing over-distension, most often by indwelling urethral catheterisation (IDC) to allow continuous drainage until postinjury diuresis resolves, usually at 7-10 days [9]. Although ongoing management may still require IDC or suprapubic catheterisation (SPC) for some patients, it is generally 
recognised that, during inpatient stay, intermittent catheterisation (IC) is preferred since this reduces the risk of UTI [10-12]. While IDC is associated with a high complication rate [13] and early implementation of IC ( $>10$ days post-SCI) can reduce positive urine cultures and urological complications [14], early IC administered by staff ('staff-IC') is not always practiced [14], with apparently wide variation between different spinal units in Australia and New Zealand [15].

During the sub-acute rehabilitation phase, an appropriate long-term bladder management method is selected, based on the patient's neurological function and personal preference as well as the recommendations of the medical team. Long-term options include spontaneous voiding (with or without pharmaceutical and/or surgical treatment), selfadministered IC (self-IC), SPC and ongoing IDC, with the latter considered as a last resort when other options are not clinically appropriate [16] or not supported by the patient. Alternative methods such as bladder expression, voiding using abdominal straining, triggered reflex voiding and the use of external appliances such as pads and condom catheters are usually discouraged due to associated increased risk of urological complications [17].

Despite various guidelines for bladder management practice, UTIs following SCI remain an intractable and major longterm health care burden with associated high financial and personal costs and new approaches are needed [15]. We therefore conducted a retrospective audit of inpatient bladder management and infections in WA. Our specific aims were to (1) determine the incidence of UTI during inpatient stay, (2) explore associations between inpatient UTIs and hospital length of stay (LOS), duration of initial IDC, bladder drainage method, demographics, injury level and severity and (3) determine the extent to which deviations from staff-IC protocol occurred and whether any deviations were associated with UTI. Our hypotheses were that (1) increased overall rate of UTIs is associated with longer hospital LOS, (2) UTIs in the acute phase of SCI are associated with an increased rate of subsequent UTIs during hospital admission and (3) deviations from staff-IC protocol are associated with increased risk of UTI.

\section{Methods}

In WA, acutely injured SCI patients are admitted to the 'Surgical Hospital', where the majority are managed via IDC. Once medically stable, patients are transferred to the State Rehabilitation Service ('Spinal Unit') for the remainder of their in-hospital rehabilitation, which includes specialist urodynamic bladder assessment and various bladder management protocols. In the absence of ongoing medical or surgical issues that would necessitate leaving the IDC in situ, the standard protocol is the removal of the IDC and commencement of staff-IC. Patients with minimal neurological impairment, and/or exhibiting neurological recovery such that spontaneous voiding may be expected to return, are given the opportunity to trial spontaneous voiding, with documentation of voided volume and assessment of postvoid residual by ultrasound. All patients undergo urodynamic assessment, which delineates bladder dysfunction in more detail and helps with developing long-term bladder management options. Staff-IC guidelines at the Spinal Unit are based on published recommendations [9, 16] and involve regular, complete emptying of the bladder at least every 6 hours (h) to reduce infection risk due to stasis; in addition, controlling fluid intake to minimise bladder volumes over $500 \mathrm{~mL}$ prevents over-distension and risk of upper urinary tract damage [16]. There are, however, no available data on how closely the staff-IC protocol is adhered to. Staff-IC usually continues until long-term management is implemented, preferably self-IC, otherwise SPC, or much less frequently, IDC. The standard assessment protocol for UTI involves a catheter specimen of urine (CSU) taken in the event of clinical signs and/or symptoms of UTI developing, or prior to an invasive urological procedure. In addition, at the time of this audit, during staff-IC periods CSUs were submitted each day by Spinal Unit ward nurses for microscopy, culture and susceptibility testing.

A retrospective audit was performed on all new acute adult traumatic SCI admissions to the Spinal Unit from 1 January 2015 to 28 February 2017 inclusive, with data collected from the beginning of each patient's acute admission at the Surgical Hospital, until discharge from the Spinal Unit. A retrospective design allowed accurate UTI diagnosis, since consideration could be given to UTI clinical signs and symptoms, concurrent illnesses, diagnosis by the treating doctor and final health outcomes. A list of traumatic SCI patients hospitalised during the audited period was obtained from the Spinal Unit $(n=93)$. Patients were excluded if medical records could not be located, the SCI and initial medical management occurred outside WA, or a previous SCI had been incurred.

The following data (Supplementary Data 1 lists full definitions) were collected from the hospital electronic database 'iSoft Clinical Manager' and patient medical records, de-identified (patient name and hospital number replaced by secure study code) and recorded in Research Electronic Database Capture (REDCap), a secure, webbased application designed to support data collection for research studies: demographics; mode and date of injury; neurological status; bladder management (IDC, staff-IC, self-IC, SPC, and spontaneous voiding SV) including temporary interruptions/changes to that management; renal ultrasound; LOS at each hospital; urinary infection events including bacteriuria (urine sample with positive culture growth $\geq 10^{4} \mathrm{CFU} / \mathrm{mL}$ of $\geq 1$ bacterial species); and UTI 
(bacteriuria with the presence of symptomatology [body temperature $>38^{\circ} \mathrm{C}$, rigors, altered mental state, malaise/ lethargy, flank pain, costovertebral angle tenderness, acute haematuria, pelvic discomfort, dysuria, urgent or frequent urination, suprapubic pain or tenderness, increased spasticity, autonomic dysreflexia, and a sense of unease] as defined by the Infectious Diseases Society of America's 2009 International Clinical Practice Guidelines) [11].

We also examined paper-based fluid balance charts, archived specifically for this project, that were maintained by nurses for patients managed via staff-IC to calculate the timing of ICs (Supplementary Data 1 show full details). Intervals of $\leq 6 \mathrm{~h}$ between ICs and maintaining volumes $<$ $500 \mathrm{~mL}$ are considered 'best practice' [9, 18], whereas intervals of $\geq 8 \mathrm{~h}$ between ICs and IC volumes $>800 \mathrm{~mL}$ are considered clinically meaningful protocol deviations by clinicians at the Spinal Unit.

\section{Statistical methods}

Summary statistics for demographics and management practices are described through mean and standard deviation for variables with reasonably symmetric distributions, and through median and interquartile ranges otherwise (i.e. for continuous variables, and counts and percentages for categorical variables).

The overall incidence, and multi-resistant organism (MRO) rates, of bacteriuria and UTIs were calculated as the number of infection starts divided by the exposure, presented as a rate per 100 days. The exposure for UTI is the number of hospitalisation days, while the exposure for asymptomatic bacteriuria $(\mathrm{AB})$ is the number of full days of staff-IC (not including interruptions). In addition, the overall UTI incidence during each phase of bladder management and at each hospital site was reported.

In order to explore factors associated with UTI and hospital LOS, modelling was undertaken using the following dependent variables: the occurrence of having at least one infection, infection rate, time to first infection and hospital LOS. In each model, the following explanatory (independent) variables were chosen based on clinical advice: gender, age, mode of injury, pre-existing conditions (musculoskeletal disease, cardiovascular disease, nervous system disorder, psychiatric disorder, ophthalmological, infectious disease, other, and none), time to initial catheter insertion (h), initial IDC duration (days), discharge neurological level and discharge AIS (American Spinal Injury Association Impairment Scale) status. In addition, infection rate modelling included time to first infection and proportion of time in the Surgical Hospital as further potential explanatory variables; while hospital LOS considered the occurrence of at least one infection as an additional explanatory variable.
Logistic regression was used to model the occurrence of at least one infection, based on all individuals. Poisson regression was used to model infection rate for those with at least one infection and the model included an offset (log of the number of days in hospital). A Cox proportional hazards model was used to model time to first infection for those with at least one infection and negative binomial regression was used to model hospital LOS (all individuals). Poisson regression was used to assess whether the infection rates were dependant on the bladder management with an offset (log of the exposure time on each bladder management type) included in the model.

As for other similar studies [19-21], backwards selection was used in all modelling, since this allowed consideration of all clinically relevant explanatory variables and the identification of those with significant associations. Significance was set at the 5\% level and data were analysed using the $\mathrm{R}$ environment [22].

To assess the impact of deviations from protocol on infection during periods of staff-IC (yes/no), modelling was carried out using logistic regression. Deviations (independent variables) considered were bladder volumes $>800 \mathrm{~mL}$, IC intervals $\geq 8 \mathrm{~h}$ and IC protocol interruptions that occurred in the week prior to an UTI.

\section{Results}

Ninety-three patient records were screened for eligibility with 70 used to examine bladder management and UTIs throughout Surgical Hospital and Spinal Unit admission (Fig. 1). One patient, admitted for 648 days, had not completed inpatient admission at the cessation of data collection. In addition, 38/70 had staff-IC for which we examined adherence to protocol (Fig. 1).

Patient characteristics, bladder management and outcomes including demographics, pre-existing medical conditions, UTIs, final bladder management, and LOS are shown $(n=70$, Table 1$)$. The median time to initial catheter insertion was 4 (interquartile range, IQR, 4.2) hours and the median initial IDC duration was 21.5 (IQR 21.0) days (Table 1, Fig. 2). Latex IDCs were predominantly used, and silicone catheters when prolonged IDC was required. The median hospital LOS was 15.2 (IQR 11.8) days at the Surgical Hospital, 78.5 (65.3) days at the Spinal Unit and 97.6 (IQR 74.4) days overall.

Median and IQRs for hospital (Surgical, Spinal Unit and total) LOS and duration of IDC are also shown for patients according to neurological status at hospital discharge $(n=$ 70, Table 2). Bladder management for each patient was 'mapped' over time, including management changes and staff-IC interruptions ( $n=70$, Fig. 2, which also indicates when UTIs occurred). 


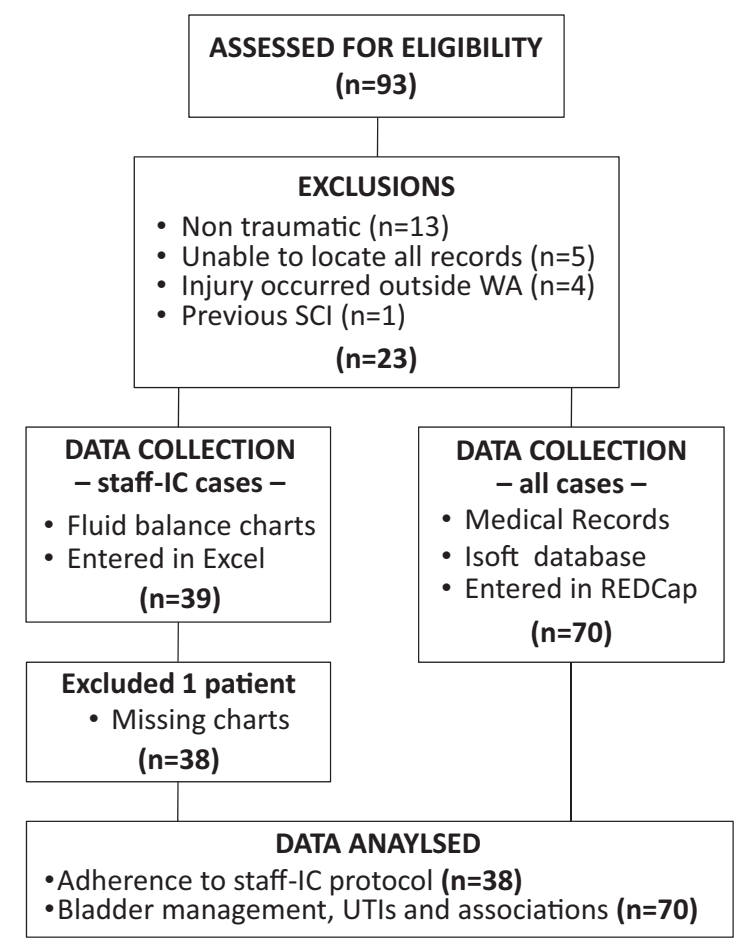

Fig. 1 Consolidated reporting of trials (CONSORT) flowchart

\section{AIM 1, incidence of UTI throughout hospital admission}

Forty-three/seventy patients (61.4\%) experienced one or more UTIs during their admission, while 27 (38.6\%) did not have any symptomatic infections. The rate of UTI was 1.1 starts per 100 days, with 88 episodes in total. Sixteen occurred at the Surgical Hospital and 76 at the Spinal Unit; however, both sites had the same incidence rate (1.1 starts per 100 days). The instances occurred during the four bladder management strategies, namely: IDC (36 instances, incidence rate 1.3 starts per 100 days), staff-IC (31 instances, incidence rate 2.0), self-IC (15 instances, incidence rate 0.8 ) and spontaneous voiding (6 instances, incidence rate 0.5 ).

Escherichia coli, Klebsiella, Enterococcus and Enterobacter were the most common bacteria reported, both in monitored urine samples taken during periods of staff-IC, and in samples taken to confirm UTI throughout admission (see Table 3 for full list of reported bacteria). AB incidence for those undergoing staff-IC (38 patients) was 2.6 infection starts per 100 days of staff-IC. Two of the episodes were caused by MROs. The rate of UTI by MRO across the whole cohort $(n=70)$ was 0.1 starts per 100 days; there were just five instances in total across the study period, three caused by methicillin-resistant Staphylococcus aureus (MRSA, all reported by the one patient) and two caused by extendedspectrum beta-lactamase-producing Enterobacteriaceae.
The overall median hospital LOS was 97.6 days. Multivariate statistical modelling of LOS showed that having one or more UTI was associated with a LOS that was 1.40 times longer than if patients were UTI-free $(95 \%$ confidence interval (CI) $1.2-9.9, p$-value $<0.001$ ); a complete injury was associated with a LOS 1.3 times longer than if patients had incomplete injuries (95\%CI 1.1-1.6, $p$-value 0.007); a pre-existing nervous system disorder was associated with a LOS 1.7 times longer (95\%CI 1.2-2.5, $p$-value 0.007); and a longer duration of initial IDC was associated with an increase LOS (rate ratio (RR) for a one standard deviation increase $1.4,95 \% \mathrm{CI} 1.3-1.6, p$-value $<0.001$ ).

\section{AIM 2, factors associated with UTI}

Multivariate statistical modelling $(n=70)$ showed that being female was associated with having at least one UTI (odds ratio (OR): 6.1, 95\%CI 1.2-44.0, $p$-value 0.028); as did being AIS complete (OR: 5.3, 95\%CI 1.3-21.1, $p$-value 0.019).

Considering the $43 / 70$ patients (61.4\%) who had $\geq 1$ UTI, the median time to first UTI was 25 (IQR: 27) days postSCI. Statistical modelling showed a shorter time to first infection was the only factor significantly associated with a higher subsequent infection rate (for a one standard deviation [30.7 days] shorter time, RR: 1.3, 95\%CI 1.0-1.7, $p$ value $=0.039)($ Supplementary Data 2$)$. Further modelling investigating predictors of time to first UTI showed a longer duration of initial IDC was associated with a shorter time to first infection (hazard ratio, for a one standard deviation [45.0 days] longer duration: $0.7,95 \% \mathrm{CI} 0.5-1.0, p$-value $0.044)$.

\section{AIM 3, adherence to staff-IC protocol}

For the $38 / 70$ patients managed for a period via staff-IC, the median length of the period was 22.3 (IQR: 49.0) days (Table 1). Polyvinyl catheters were used during this period, as well as for self-IC (data not shown). Fluid balance charts showed there were 5621 instances of staff-IC with 5585 intervals between staff-ICs and that $86 \%$ were conducted at $\leq 6 \mathrm{~h}$ apart and $96 \%$ at $<8 \mathrm{~h}$ apart (Table 3; Supplementary Data 3). The overall mean interval between staff-ICs was 5.9 h (IQR: 1.0) hours (rounded to 6; Table 1). Seventy four percent of IC volumes were $500 \mathrm{~mL}$ or less; $26 \%$ exceeded $500 \mathrm{~mL}$ and $4 \%$ exceeded $800 \mathrm{~mL}$ (Table 3). Modelling of associations between UTI and staff-IC protocol deviations found that a high bladder volume $(>800 \mathrm{~mL})$ was associated with an increased UTI rate during the following week (OR: 1.6, 95\%CI 1.1-2.3, $p$-value 0.009); and an interruption to the staff-IC protocol was associated with an increased UTI rate during the following week (OR: 3.9, 95\%CI 2.6-5.9, $p$ value $<0.001)$. 
Table 1 Summary of all $(n=70)$ patients: demographics and characteristics as well as bladder management and UTIs

\begin{tabular}{|c|c|c|c|c|c|}
\hline \multicolumn{3}{|c|}{ Patient demographics and characteristics } & \multicolumn{3}{|c|}{ Bladder management and symptomatic urinary tract infections } \\
\hline & Median & IQR & & Median & $\mathrm{IQR}$ \\
\hline \multirow[t]{2}{*}{ Age } & 46 & 31 & Time to initial IDC (hours) & 4 & 4 \\
\hline & Frequency & Percentage & & & \\
\hline Gender & & & Initial IDC duration (days) & 22 & 21 \\
\hline Male & 55 & 79 & & & \\
\hline Female & 15 & 21 & Length of staff-IC period (days) & 20 & 49 \\
\hline \multicolumn{6}{|c|}{ Pre-existing medical conditions } \\
\hline Cardiovascular disease & 21 & 30 & $\begin{array}{l}\text { Time to first UTI for patients with } \geq 1 \\
\text { (days) }\end{array}$ & 25 & 27 \\
\hline Infectious disease & 1 & 1 & & & \\
\hline Musculoskeletal disease & 8 & 11 & Interval between staff-ICs (hours) & 6 & $0^{*}$ \\
\hline Nervous system disorder & 4 & 6 & & & \\
\hline Ophthalmological & 5 & 7 & Length of stay (days) & & \\
\hline Psychiatric disorder & 15 & 21 & Surgical hospital & 15 & 12 \\
\hline Other & 29 & 41 & Spinal Unit & 79 & 65 \\
\hline None & 19 & 27 & Overall & 98 & 74 \\
\hline Mode of injury & & & & Frequency & Percentage \\
\hline Fall & 23 & 33 & Had staff-IC & & \\
\hline Motor vehicle accident & 30 & 43 & Yes & 38 & 54 \\
\hline$M V A \multimap c a r$ & 17 & 24 & No & 32 & 46 \\
\hline MVA-motorcycle & 13 & 19 & Had $\geq 1$ UTI & & \\
\hline Water-related & 6 & 8 & Yes & 43 & 61 \\
\hline Other & 11 & 16 & No & 27 & 39 \\
\hline \multicolumn{6}{|l|}{ Discharge AIS } \\
\hline Complete & 19 & 21 & Long-term bladder management & & \\
\hline Incomplete & 51 & 73 & Carer-IC & 1 & 1 \\
\hline Discharge level & & & IDC & 3 & 4 \\
\hline $\mathrm{C} 1-\mathrm{C} 4$ & 21 & 30 & Self-IC & 25 & 36 \\
\hline $\mathrm{C} 5-\mathrm{C} 8$ & 18 & 26 & SPC & 10 & 14 \\
\hline T1-T6 & 11 & 16 & SV & 29 & 42 \\
\hline T7-T12 & 10 & 14 & Unknown & 2 & 3 \\
\hline L1-L5 & 10 & 14 & & & \\
\hline
\end{tabular}

NB: percentages for pre-existing medical conditions sum to $>100 \%$ since some patients had $>1$

*0 is shown because $>$ half of intervals between staff-ICs were exactly $6 \mathrm{~h}$

\section{Discussion}

\section{Early bladder management $(n=70)$}

Reducing UTI incidence following SCI is important from patient, clinical and economic perspectives. Although demonstration of association does not indicate causation, our findings suggest that early removal of IDCs may be beneficial. The median initial IDC duration for our cohort was 21.5 days (average: 37; range: 6-234 days). IDC duration is linked to LOS at the Surgical Hospital (median: 15 days, average: 21 days), where patients are not progressed to IC. LOS at the Surgical Hospital was high for some patients, particularly those with complete tetraplegia (median: 40.4 days, average: 54 days). The implementation of a staff-IC protocol at the Surgical Hospital may benefit some patients, particularly those with complete tetraplegia and long LOS.
Our finding that a shorter time to first UTI is associated with a higher subsequent infection rate may be of interest. We also demonstrate that having one or more UTIs is associated with a hospital stay 1.4 times longer than for those who are UTI-free $(p<0.001)$ [23]. Female gender and complete injury were anticipated associations [24], since females are more susceptible to UTIs in the general population [25] and patients with severe injuries are often very unwell acutely, with neurotrauma inducing immune deficiency, a higher degree of disturbance to bladder function and increased susceptibility to infection [26].

\section{Adherence to staff-IC protocol and UTI $(n=38)$}

The value of IC in reducing chronic urogenital complications including UTIs is known [10-12] and was established as a 6-hourly regimen previously $[18,27]$. Clinical guidelines continue to highlight minimisation of large volumes to 


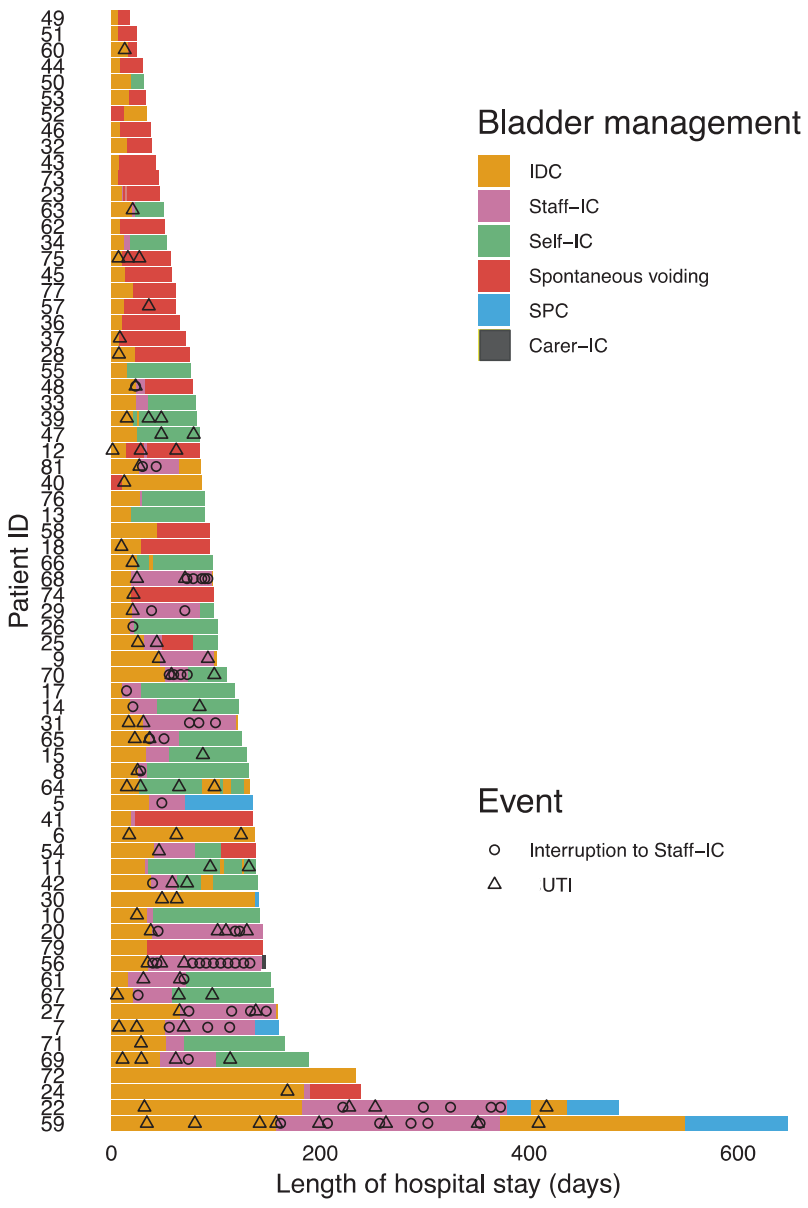

Fig. 2 Bladder management for each patient $(n=70)$ over time with the interruptions to staff-IC and UTIs marked at their starting times

maintain safe pressures during bladder filling and prevent incontinence between catheterisations, advising 6-hourly (or more frequent) ICs, careful monitoring of fluid intake/output and regular bladder ultrasound scanning [9]. Despite showing adherence to a 6-hourly regimen for staff-IC in the majority of cases, approximately one quarter of IC volumes exceeded $500 \mathrm{~mL}$. We noted that minimising very high volumes $(>800 \mathrm{~mL})$ relies on good patient compliance, with medical records indicating reasons for significantly delayed staff-ICs that were beyond the control of attending nurses including: patients refusing to be catheterised, being absent at scheduled times following day/weekend leave or having just voided spontaneously. In addition to fluid intake/output, other factors may contribute to higher than ideal bladder volumes, including fluctuations in urine output, which have anecdotally been reported to increase during mobilisation [28], and normalisation of diurnal antidiuretic hormone secretion and urine output, which is disrupted after SCI [29].

High volumes are thought to over-distend the bladder, thereby damaging the urothelial barrier, making it more susceptible to leakage and infection [30]. Our data showed that both interruptions to staff-IC and high volumes were associated with higher UTI rates in the following week, supporting a previous study of 302 out-patients, ranging from normal to severe disability, in which high catheterisation volumes were associated with UTIs [31].

UTI incidence did not vary according to staff- or self- IC management. A higher UTI incidence during self-IC might be anticipated since nurses are trained to use a sterile, notouch technique, whereas patients are new to self-IC and use a clean, rather than sterile, technique. Possible explanations for UTI incidence during staff-IC periods being similar to that during self-IC are lapses in nursing practice standards, for example, when less experienced nurses conduct staffICs with imperfect technique, and greater cross infection compared with self-IC. Indeed others have shown that cross infection is reduced when IC during hospitalisation is conducted by the patients themselves, or by a small dedicated catheter team, compared with when administered by attending nurses [32].

\section{Implications for practice}

Limiting IDC duration and progressing to staff-IC when safe to do so appear to be associated with reduced early UTIs, supporting clinical guidelines [9, 16, 33]. Close adherence to 6-hourly staff-IC demonstrated that this protocol is practicable, however a number of volumes $>500$ $\mathrm{mL}$ indicates that closer monitoring of fluid balance may be required. The associations between bladder volumes $>800$ $\mathrm{mL}$ and interruptions to staff-IC with increased UTIs in the following week reinforces the importance of protocol adherence. However, exceptions to the protocol are inevitable, for example, IDCs were temporarily inserted to enable patients to go on leave (23 instances), when patients were medically unwell and/or required IV fluids (10 instances), had haematuria or required a bladder washout (10 instances), had high IC volumes ( 2 instances) and when medical procedures were required (15 instances).

\section{Study limitations}

Compared with other reports [34, 35], the number of patients in this exploratory study was relatively small $(n=$ 70) and we recognise that the associations described should be treated with caution. The selection of appropriate variables to incorporate in statistical analyses of observational data is challenging. Our approach used expert knowledge to identify clinically relevant explanatory variables, together with regression modelling with backwards selection (which removes variables one by one until only variables with an alpha above a pre-specified threshold remain), to identify those variables with associations. Backward selection is preferred when there is a moderate number of explanatory 


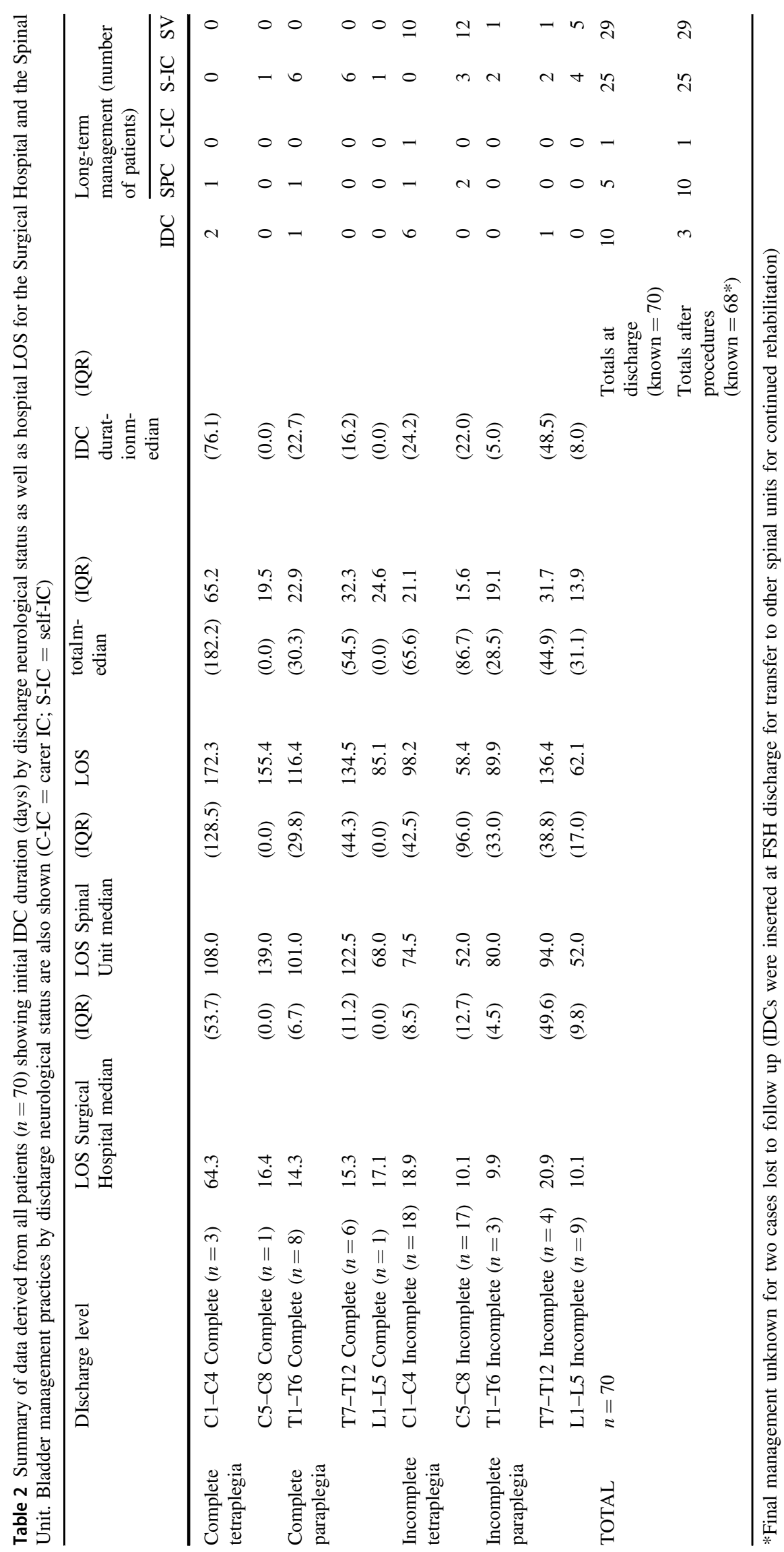


Table 3 Summary of data derived from staff-IC patients $(n=38 / 70)$

\begin{tabular}{|c|c|c|}
\hline Time between staff-ICs & Count & Percentage \\
\hline Six hours or less & 4788 & 86 \\
\hline Less than 8 hours & 5366 & 96 \\
\hline Eight hours or more & 218 & 4 \\
\hline Total & 5584 & 100 \\
\hline \multicolumn{3}{|c|}{ Urine volume drained at each catheter } \\
\hline $500 \mathrm{~mL}$ or less & 4167 & 74 \\
\hline More than $500 \mathrm{~mL}$ & 1454 & 26 \\
\hline More than $500-800 \mathrm{~mL}$ & 1259 & 22 \\
\hline $800 \mathrm{~mL}$ or less & 5426 & 96 \\
\hline More than $800 \mathrm{~mL}$ & 195 & 4 \\
\hline Total & 5621 & 100 \\
\hline \multicolumn{3}{|l|}{ Urine cultures } \\
\hline Species & $\begin{array}{l}\text { Asymptomatic bacteriuria: staff- } \\
\text { IC periods (number) }\end{array}$ & $\begin{array}{l}\text { Symptomatic UTI: throughout } \\
\text { study (number) }\end{array}$ \\
\hline Escherichia coli & 8 & 38 \\
\hline Klebsiella spp. & 13 & 30 \\
\hline Enterobacter spp. & 5 & 14 \\
\hline Enterococcus spp. & 12 & 13 \\
\hline Pseudomonas aeruginosa & 4 & 8 \\
\hline Proteusm mirabilis & 0 & 5 \\
\hline Serratia spp. & 0 & 3 \\
\hline Acinetobacter baumanni & 0 & 1 \\
\hline Citrobacter koseri & 1 & 2 \\
\hline Citrobacter Non koseri & 0 & 0 \\
\hline Morganella spp. & 1 & 0 \\
\hline Candida albicans & 0 & 0 \\
\hline Enterococcus faecium & 0 & 1 \\
\hline $\begin{array}{l}\text { Methicillin-Resistant } \\
\text { Staphylococcus aureus }\end{array}$ & 1 & 3 \\
\hline Other & 6 & 5 \\
\hline
\end{tabular}

'Count' refers to numbers of entries in the fluid balance charts. Numbers of times bacterial and fungal species were reported from urine cultures for symptomatic urinary tract infections and asymptomatic bacteriuria are also given. Time between staff-ICs and urine volumes collected were calculated from fluid balance charts

variables of which some need to be removed [36], as was the case in this study. Using directed acyclic graphs (DAGs) is an alternative modelling approach, particularly in larger studies where it is more reasonable to investigate causality. In brief, a DAG is a modelling approach and graphical tool that provides a way to visually represent the variables such as exposure (for example, time in hospital), outcome, confounders, bias and causation (via 'edges' in the DAG) [37]. Future studies should be mindful of the range of possibilities for analysing observational data.

Due to urology reports on urodynamic assessments not being available during the data collection period for this study, we could not evaluate whether, for example, abnormal bladder compliance or capacity, detrusor function, maximum pressure and/or leak point pressure are associated with UTI. However, considering neurogenic detrusor overactivity increases UTI risk [38] and treatment reducing this overactivity has been shown to reduce UTI incidence [39], urodynamic assessment should be included in future prospective studies evaluating UTI following SCI.

Catheter type for IC may also have an impact on UTI risk, although high-quality published evidence on this topic is conflicting [35, 40-42]. In the current study, standard polyvinyl catheters were consistently used across staff-IC and self-IC periods, and so it was not possible to analyse associations between UTI rates and catheter type.

Findings from this single-site study are relevant only to other sites where bladder management is similar. Although a recent survey indicated differences in bladder 
management at Australian and New Zealand sites [15], these are anecdotal. The current detailed study may provide the basis for larger multi-centre prospective studies to examine possible relationships between, for example, time to first UTI and subsequent UTI rate.

\section{Data archiving}

The datasets generated and/or analysed during the current study will be made available in The University of Western Australia's research repository; https://research-repository. uwa.edu.au/en/datasets.

Acknowledgements We thank Tony Smith for assistance on REDCap database design and Dr Duncan McLellan, Fiona Stanley Hospital, for discussions during the course of this study.

Funding Neurotrauma Research Program, Western Australia; The University of Western Australia.

Author contributions LMG and SAD: grant writing, study design, project oversight, data collection, manuscript, table and figure preparation. GKK: data collection, intellectual input on manuscript, tables and figures. AR and KM: statistical plans, modelling and analysis, intellectual input on manuscript, tables and figures. $\mathrm{PB}, \mathrm{AW}, \mathrm{CH}, \mathrm{JT}, \mathrm{JAB}$ and $\mathrm{MR}$ : intellectual input on study design, manuscript, tables and figures.

\section{Compliance with ethical standards}

Conflict of interest The authors declare that they have no conflict of interest.

Statement of ethics The South Metropolitan Health Service Human Research Ethics Committee provided ethical approval (number 16148) for the project. We certify that all applicable institutional and governmental regulations concerning the ethical use of human volunteers/animals were followed during the course of this research.

Publisher's note: Springer Nature remains neutral with regard to jurisdictional claims in published maps and institutional affiliations.

\section{References}

1. Vigil HR, Hickling DR. Urinary tract infection in the neurogenic bladder. Transl Androl Urol. 2016;5:72-87.

2. DeJong G, Tian W, Hsieh C-H, Junn C, Karam C, Ballard PH. et al. Rehospitalization in the first year of traumatic spinal cord injury after discharge from medical rehabilitation. Arch Phys Med Rehabil. 2013;94:S87-97.

3. Gabbe BJ, Nunn A. Profile and costs of secondary conditions resulting in emergency department presentations and readmission to hospital following traumatic spinal cord injury. Injury. 2016;47:1847-55.

4. Cardenas DD, Hoffman JM, Kirshblum S, McKinley W. Etiology and incidence of rehospitalization after traumatic spinal cord injury: a multicenter analysis. Arch Phys Med Rehabil. 2004;85:1757-63.

5. Siroky MB. Pathogenesis of bacteriuria and infection in the spinal cord injured patient. Am J Med. 2002;113:67S-79S.
6. Watts A. An audit of readmissions of people with spinal cord injury to a major metropolitan teaching hospital in Perth, Western Australia. Royal Perth Hospital. 2012. Unpublished.

7. Simpson LA, Eng JJ, Hsieh JT, Wolfe DL. Spinal cord injury rehabilitation evidence Scire research $\mathrm{T}$. The health and life priorities of individuals with spinal cord injury: a systematic review. J Neurotrauma. 2012;29:1548-55.

8. Lee JS, Kim SW, Jee SH, Kim JC, Choi JB, Cho SY. et al. Factors affecting quality of life among spinal cord injury patients in Korea. Int Neurourol J. 2016;20:316-20.

9. Middleton J, Ramakrishnan K, Cameron I, Innovation NAfC. Management of the neurogenic bladder for adults with spinal cord injuries. 3rd ed. Agency for Clinical Innovation, NSW Government, Chatswood, NSW; 2014. p 1-15.

10. Everaert K, Lumen N, Kerckhaert W, Willaert P, van Driel M. Urinary tract infections in spinal cord injury: prevention and treatment guidelines. Acta Clin Belg. 2009;64:335-40.

11. Hooton TM, Bradley SF, Cardenas DD, Colgan R, Geerlings SE, Rice JC, et al. Diagnosis, prevention, and treatment of catheterassociated urinary tract infection in adults: 2009 International Clinical Practice Guidelines from the Infectious Diseases Society of America. Clin Infect Dis. 2010;50:625-63.

12. Ryu KH, Kim YB, Yang SO, Lee JK, Jung TY. Results of urine culture and antimicrobial sensitivity tests according to the voiding method over 10 years in patients with spinal cord injury. Korean J Urol. 2011;52:345-9.

13. Nicolle LE. Urinary catheter-associated infections. Infect Dis Clin N Am. 2012;26:13-27.

14. Zermann D, Wunderlich H, Derry F, Schroder S, Schubert J. Audit of early bladder management complications after spinal cord injury in first-treating hospitals. Eur Urol. 2000;37:156-60.

15. Goodwin DM, Brock J, Dunlop S, Goodes L, Middleton J, Nunn A, et al. Optimal bladder management following spinal cord injury: evidence, practice and a cooperative approach driving future directions in Australia. Arch Phys Med Rehabil. 2018;99:2118-21.

16. Wright B, Bragge P. Spinal cord injury bladder care recommendations matrix. BehaviourWorks Australia, Monash Sustainability Institute, Monash University, VIC, Nov 2015, p. 1-30.

17. Blok B, Pannek J, Castro-Diaz D, del Popolo G, Groen J, Hamid R. et al. EAU Guidelines on Neuro-Urology. European Association of Urology, Arnhem, The Netherlands; 2019. p. 1-52. http:// uroweb.org/guidelines/compilations-of-all-guidelines/.

18. Pearman JW. Urological follow-up of 99 spinal injured patients initially managed by intermittent catherisation. $\mathrm{Br} \mathrm{J}$ Urol. 1976;48:297-310.

19. Albaramki J, Abdelghani T, Dalaeen A, Ahmad FK, Alassaf A, Odeh R, et al. Urinary tract infection caused by ESBL- producing bacteria: risk factors and antibiotic resistance. Pediatr Int. 2019. https://doi.org/10.1111/ped.13911. [Epub ahead of print]

20. Bloomfield MG, Carmichael AJ, Gkrania-Klotsas E. Mortality in Clostridium difficile infection: a prospective analysis of risk predictors. Eur J Gastroenterol Hepatol. 2013;25:700-5.

21. Moritz ED, Smith TC. Risk of Staphylococcus aureus carriage in childcare employees. Epidemiol Infect. 2013;141:1975-82.

22. Team RC. R: a language and environment for statistical computing. Vienna, Austria: R Project for Statistical Computing; 2017.

23. Street JT, Noonan VK, Cheung A, Fisher CG, Dvorak MF. Incidence of acute care adverse events and long-term healthrelated quality of life in patients with TSCI. Spine J. 2015;15:923-32.

24. Marion TE, Rivers CS, Kurban D, Cheng CL, Fallah N, Batke J, et al. Previously identified common post-injury adverse events in traumatic spinal cord injury-validation of existing literature and relation to selected potentially modifiable comorbidities: a 
prospective Canadian cohort study. J Neurotrauma. 2017; 34:2883-91.

25. Foxman B. Epidemiology of urinary tract infections: incidence, morbidity, and economic costs. Am J Med. 2002;113 Suppl $1 \mathrm{~A}: 5 \mathrm{~s}-13 \mathrm{~s}$

26. Meisel C, Schwab JM, Prass K, Meisel A, Dirnagl U. Central nervous system injury-induced immune deficiency syndrome. Nat Rev Neurosci. 2005;6:775-86.

27. Guttmann L, Frankel H. The value of intermittent catheterisation in the early management of traumatic paraplegia and tetraplegia. Paraplegia. 1966;4:63-84.

28. Angeli CA, Edgerton VR, Gerasimenko YP, Harkema SJ. Altering spinal cord excitability enables voluntary movements after chronic complete paralysis in humans. Brain. 2014;137:1394-409.

29. Kilinc S, Akman M, Levendoglu F, Ozker R. Diurnal variation of antidiuretic hormone and urinary output in spinal cord injury. Spinal Cord. 1999;37:332-5.

30. Vasudeva P, Madersbacher H. Factors implicated in pathogenesis of urinary tract infections in neurogenic bladders: some revered, few forgotten, others ignored. Neurourol Urodyn. 2014; 33:95-100.

31. Bakke A, Vollset SE, Høisæter PÅ, Irgens LM. Physical Complications in patients treated with clean intermittent catheterization. Scand J Urol Nephrol. 1993;27:55-61.

32. Wyndaele JJ, De Taeye N. Early intermittent self-catheterisation after spinal cord injury. Paraplegia. 1990;28:76-80.

33. Stohrer M, Blok B, Castro-Diaz D, Chartier-Kastler E, Del Popolo $\mathrm{G}$, Kramer G, et al. EAU guidelines on neurogenic lower urinary tract dysfunction. Eur Urol. 2009;56:81-8.

34. Escalarin De Ruz A, Garcia Leoni E, Herruzo Cabrera R. Epidemiology and risk factors for urinary tract infection in patients with spinal cord injury. J Urol. 2000;164:1285-9.

35. Cardenas DD, Moore KN, Dannels-McClure A, Scelza WM, Graves DE, Brooks M, et al. Intermittent catheterization with a hydrophilic-coated catheter delays urinary tract infections in acute spinal cord injury: a prospective, randomized, multicenter trial. PM R. 2011;3:408-17.

36. Martínez MN, Suárez E, Pérez CM. Chapter 5: selection of variables in a multiple linear regression model. In: Applications of regression models in epidemiology. 1st ed. John Wiley \& Sons, Inc., Hoboken, New Jersey; 2017.

37. Williams TC, Bach CC, Matthiesen NB, Henriksen TB, Gagliardi L. Directed acyclic graphs: a tool for causal studies in paediatrics. Pedia Res. 2018;84:487-93.

38. Salameh A, Mohajer MA, D'arouchihe RO. Prevention of urinary tract infections in patients with spinal cord injury. CMAJ. 2015;187:807-11.

39. Game X, Castel-Lacanal E, Bentaleb Y, Thiry-Escudie I, De Boissezon X, Malavaud B, et al. Botulinum toxin A detrusor injections in patients with neurogenic detrusor overactivity significantly decrease the incidence of symptomatic urinary tract infections. Eur Urol. 2008;53:613-8.

40. De Ridder DJ, Everaert K, Fernandez LG, Valero JV, Duran AB, Abrisqueta ML, et al. Intermittent catheterisation with hydrophilic-coated catheters (SpeediCath) reduces the risk of clinical urinary tract infection in spinal cord injured patients: a prospective randomised parallel comparative trial. Eur Urol. 2005;48:991-5.

41. Li L, Ye W, Ruan H, Yang B, Zhang S, Li L. Impact of hydrophilic catheters on urinary tract infections in people with spinal cord injury: systematic review and meta-analysis of randomized controlled trials. Arch Phys Med Rehabil. 2013;94:782-7.

42. Bermingham SL, Hodgkinson S, Wright S, Hayter E, Spinks J, Pellowe C. Intermittent self catheterisation with hydrophilic, gel reservoir, and non-coated catheters: a systematic review and cost effectiveness analysis. Brit Med J. 2013;346:e8639.

\section{Affiliations}

\section{Louise M. Goodes ${ }^{1} \cdot$ Gabrielle K. King ${ }^{1} \cdot$ Alethea Rea ${ }^{2} \cdot$ Kevin Murray $\mathbb{D}^{3} \cdot$ Peter Boan ${ }^{4} \cdot$ Anne Watts $^{5}$. Jen Bardsley ${ }^{5}$.}

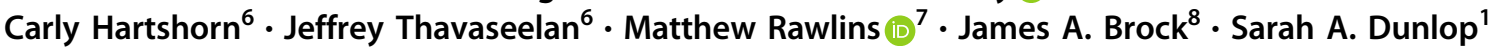

1 School of Biological Sciences, The University of Western Australia, Crawley, WA, Australia

2 Centre for Applied Statistics, The University of Western Australia, Crawley, WA, Australia

3 School of Population and Global Health, The University of Western Australia, Crawley, WA, Australia

4 Departments of Microbiology and Infectious Diseases, Fiona Stanley Hospital and PathWest Laboratory Medicine, Murdoch, WA, Australia
5 State Rehabilitation Service, Fiona Stanley Hospital, Murdoch, WA, Australia

6 Perth Urology Clinic, Murdoch, WA, Australia

7 Department of Pharmacy, Fiona Stanley Hospital, Murdoch, WA, Australia

8 Department of Anatomy and Neuroscience, University of Melbourne, Parkville, VIC, Australia 